\title{
THE TAURID COMPLEX: GIANT COMET ORIGIN?
}

\author{
D.I.STEEL ${ }^{1,2}$, D.J.ASHER ${ }^{2}$ and S.V.M.CLUBE ${ }^{2}$ \\ (1) Department of Physics and Mathematical Physics \\ University of Adelaide, G.P.O.Box 498, Adelaide, SA 5001, Australia \\ (2) Department of Physics, University of Oxford \\ Keble Road, Oxford, OX1 3RH, United Kingdom
}

\begin{abstract}
The formation and evolution of the Taurid Complex of interplanetary objects is modelled on the basis of the parent being a giant comet which entered the inner solar system some time in the past 10,000-20,000 years. The orbital element distributions for the presently-observed meteor showers are discussed in terms of how these can constrain any model for the origin of the overall complex. As a baseline model we present results from the numerical integrations of fictitious meteoroids released from a comet over ten millenia, this comet having initial elements similar to those derived from a backwards integration of $\mathrm{P} /$ Encke. Large relative velocities at perihelion, above those feasible in conventional ejection scenarios, are necessary; we ascribe these to jetting of organics and other volatiles soon after release. Such a model gives a good first-order fit to the observed orbits, although additional processes (cometary splitting or asteroidal collisions) appear also to be necessary to explain the Taurids.
\end{abstract}

\section{Introduction}

The Taurid Complex (hereafter TC) of interplanetary objects consists of several nighttime (optical and radar) and daytime (radar) meteor showers, P/Comet Encke and possibly other comets, and also several Apollo-type asteroids. The TC may well be the major source of the zodiacal dust cloud and also a large fraction of the broad sporadic meteoroid complex; it also appears to contain many other large objects which have orbits intersected by the Earth during the last few days of June each year, such as the Tunguska object. We believe that there is strong evidence to link the TC with climatic variations and catastrophic events which have occurred over the past few millennia (Clube and Asher, 1990), the root cause of which is the decay of the remnant components of a giant comet which entered a small-perihelion, short-period orbit within the last few tens of thousand years. This time-scale is supported by other recent modelling of the TC (Babadzhanov et al, 1990). In order to understand past events within this framework, and to make predictions as to the future influence of the TC upon the terrestrial environment, it is necessary to explain observed TC data in terms of a consistent model of the evolution of the objects spawned by the giant comet (smaller comets, asteroids, meteoroids). In this paper we consider a large number of the meteoroid orbits which give us most of our information on the orbital distribution of the TC. We then perform numerical integrations of the orbits of fictitious meteoroids released from a model comet over ten millenia, to see whether a fit to the observed data is attainable; a more detailed analysis is given elsewhere (Steel, Asher and Clube, 1991; hereafter SAC).

A.C. Levasseur-Regourd and H. Hasegawa (eds.), Origin and Evolution of Interplanetary Dust, 327-330. C 1991 Kluwer Academic Publishers, Printed in Japan. 


\section{Observed Taurid meteors compared with model integrations}

We selected from the many thousands of orbits available from the IAU Meteor Data Center 313 meteors which we believe to be members of the TC. Of these 170 were optically-recorded, 143 radar-recorded, and of these 143, 56 were from the daytime showers around June. The selection was on the basis of radiants, times of occurrence and orbital similarity, using similar but distinct selection criteria to those used by Stohl and Porubcan (1990); for details see SAC. In Figure 1 we plot the orbital elements $a, e, q$ and $i$ (referred to Jupiter, which controls the orbital evolution of these bodies) against the nodal longitude $\Omega$ for the case of the optical Northern Taurids; 53 meteors contribute. We find, as did Stohl and Porubcan, evidence for a trend in the different elements with $\Omega$, but we also find that $a$ and $e$ appear to be correlated. This counts against the simple collision model of Whipple and Hamid (1952), since the orbits produced in a collision in the asteroid belt would have their aphelion distances nearconstant and hence $a$ and $e$ anticorrelated. Although it is possible that such collisions have contributed to the stream formation and evolution, the question arises as to whether the spread in $q$, the main reason for Whipple and Hamid's adoption of a collision model, can be explained solely by planetary perturbations.

Our provisional model is that of a large comet with small perihelion distance which entered the inner solar system about 20,000 years ago, producing meteoroids over some 10,000 years which have long since dispersed into the zodiacal cloud, and of subsequent activity within the last 10,000 years giving rise to the present appearance of the showers. The parent object is loosely based upon a backwards integration of P/Encke, and 9,000 years ago had $a=2.2 \mathrm{AU}, e=0.89$. The ejections are taken to occur at perihelion with velocities of $-2.0,-1.5,-1.0,-0.5,-0.3,-0.1,+0.1$ and $+0.3 \mathrm{~km} / \mathrm{sec}$ along the direction of motion of the parent at that point; this means that the ejections are strongly non-isotropic, but unless the semi-major axis is substantially different from the parent the orbital evolution is very similar, so there is little point in integrating objects ejected in other directions at perihelion. In addition, particles with larger positive velocities (resulting in $a>2.5 \mathrm{AU}$ ) are rapidly displaced from the TC by the effect of Jupiter, so there is no point in including these in the integrations either. The velocities and overall time-scale (less than 10,000 years) were ultimately chosen so as to fit the observed $(a, e)$ plots. For each of these eight velocities we consider nine separate ejections spaced about 1000 years apart from 9000 to 1000 years ago. These 72 orbits were then integrated forward to the present, taking into account the effect of the planets Jupiter, Saturn, the Earth and Venus. In Figure 2 we have plotted orbital element distributions for the 26 particles in this model which would have intersected the Earth's orbit within the last 4,000 years under conditions such that they would be classified as Northern Taurids; this Figure should therefore be compared with Figure 1. We note the following:

(a) A sufficiently large range in $\Omega$ has been produced by the model integrations to fit the duration of the meteor shower.

(b) Because of the choice of relative velocities there is a sufficient spread in semi-major axes, but the observational data show some larger $(a>1.5 \mathrm{AU})$ orbits at the onset of the shower (near $\Omega=180$ degrees) not seen in the model. It may be that our initial $\Omega$ for the parent was about ten degrees too high, and a better fit may be obtained in our later integrations by making such a correction.

(c) There is too small a scatter in the $e$ and $q$ plots produced by the model; more precisely, we do not see $q>0.4 \mathrm{AU}$. This may be due in part to the choice of the positive relative velocities: $+0.1 \mathrm{~km} / \mathrm{sec}$ results in the particle being injected into the $7: 2$ resonance with Jupiter, similarly ejection at $+0.3 \mathrm{~km} / \mathrm{sec}$ places a particle into the $3: 1$ 
resonance, and there is a tendency for particles in resonances not to show the increase in $q$ (i.e. decrease in $e$ ) that most particles demonstrate.

(d) In Figure 2 the particles at $\Omega<205$ degrees result from one cycle in $\omega$ of the parent, whilst those at $\Omega>205$ degrees result from the next cycle, the parent's $\omega$ having increased by 360 degrees. This may explain the existence of the two groupings seen in the observed Northern Taurids (see SAC).

(e) For the inclinations the fit to the data is reasonable although again there is not as much scatter as in the observational data.
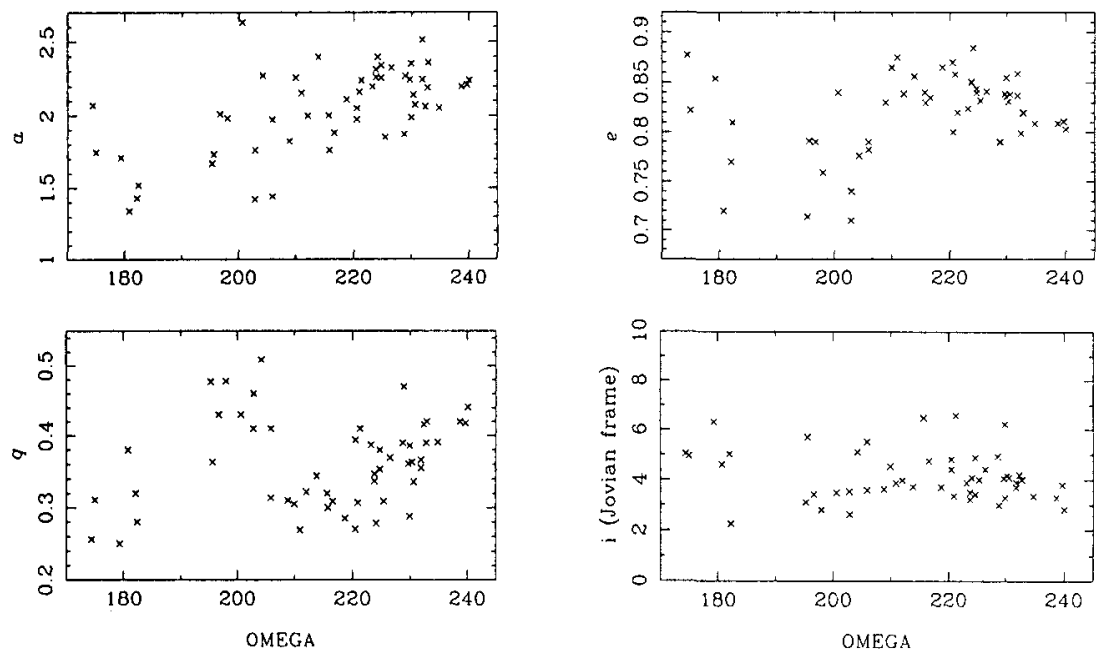

Figure 1. Orbital element distributions for 53 Northern Taurids observed in various optical meteor surveys.
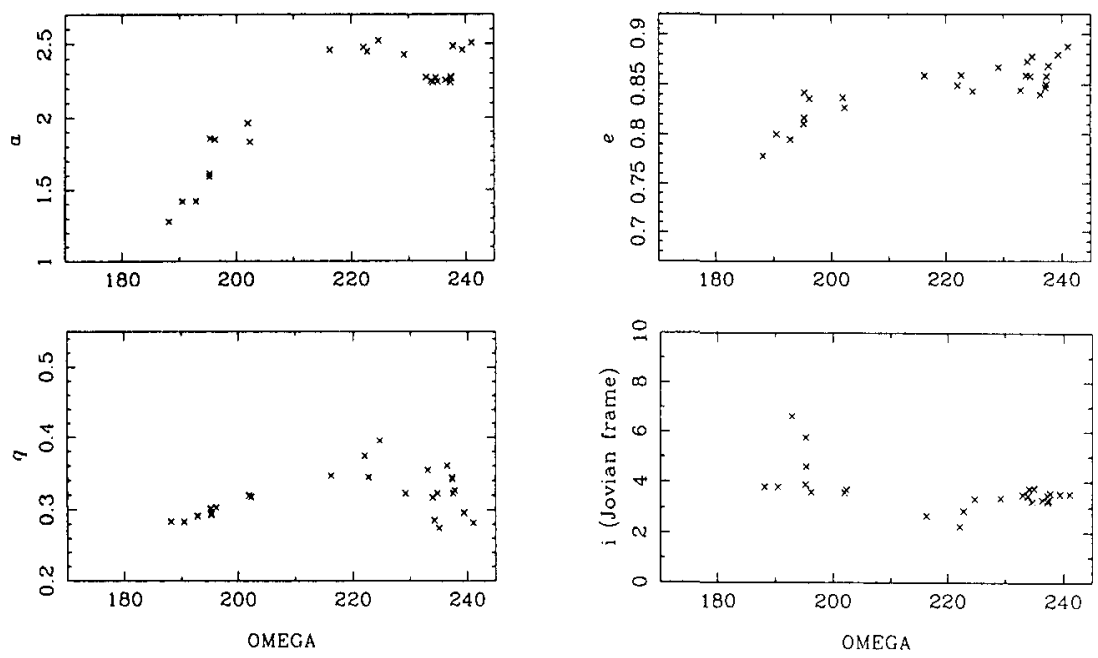

Figure 2. Model orbital element distributions for 26 meteoroids derived from a giant comet after integrations of their orbits spanning ten millenia. 


\section{Discussion}

On the basis of these preliminary integrations we believe that the provisional model, that of a giant comet which entered the inner solar system some time within the last 20,000-10,000 years and was captured into a short-period orbit with small perihelion distance $(0.15-0.25 \mathrm{AU})$, is a suitable baseline for explaining the gross phenomena observed in the TC. One drawback is that large relative velocities are required, and gravitational perturbations cannot produce these since they leave the semi-major axis largely invariant. Ejections from the cometary nucleus, with the velocity dispersion due to meteoroids being swept out by the expanding coma also cannot give such high ejection velocities: even for a comet nucleus larger than $100 \mathrm{~km}$ with a very small perihelion distance $(q<0.1 \mathrm{AU})$ the velocities tend to be below $0.1 \mathrm{~km} / \mathrm{sec}$ for $1 \mathrm{~mm}-10 \mathrm{~cm}$ meteoroids. We are thus forced to look for an alternative explanation: this is not a problem unique to the Taurids since both the Geminids and the Perseids have velocity dispersions that cannot be explained in terms of either ejection velocities from the parent or planetary perturbations. One possibility is that the dispersion is due to large amounts of organic and other volatile materials known, since the advent of the spacecraft missions to $\mathrm{P} / \mathrm{Halley}$, to exist in solid particles ejected from cometary nuclei. The jetting effect as these constituents rapidly evaporate would cause a severe alteration in the velocity of both small and large objects relative to the cometary nucleus.

In our model we do not exclude the possibility inter alia that the original comet may have been split, by collisions or otherwise, to render two or more discrete sources for meteoroid ejection: these sources may be seen now as P/Encke, several Apollo-type asteroids, and quite likely many other large bodies which are yet to be discovered, including possibly the main fragment (Clube and Asher, 1990). Since the majority of the ejecta from a comet, especially the larger masses, would remain in orbits close to that of the parent(s), we find from our integrations that there are epochs of large influx to the Earth which last for several hundred years and are spaced by several thousand years. If there were several large fragments of the original giant comet which split to form secondary sources, then such periods of enhanced meteoric activity with large body (Tunguska-sized) impacts may be interwoven in time. These are thought to be evidenced in the historical record (Clube and Napier, 1990).

Acknowledgements: This work was supported by the Science and Engineering Research Council and the Australian Research Council.

\section{References}

Babadzhanov, P.B., Obrubov, Yu.V. and Makhmudov, N. (1990). 'Encke meteor showers', Astron.Vestn., 24, 18-28.

Clube, S.V.M. and Asher, D.J. (1990). The evolution of proto-Encke: Dust bands, close encounters and climatic modulations', in Asteroids, Comets, Meteors III (eds. C.-I, Lagerkvist, H. Rickman, B.A. Lindblad and M. Lindgren), University of Uppsala Press, Sweden, 275-280.

Clube, S.V.M. and Napier, W.M. (1990). The Cosmic Winter, Basil Blackwells, Oxford, U.K.

Steel, D.I., Asher, D.J. and Clube, S.V.M. (1991). 'The structure and evolution of the Taurid complex', Mon. Not. Roy. Astron. Soc. (submitted).

Stohl, J. and Porubcan, V. (1990). 'Structure of the Taurid meteor complex', in Asteroids, Comets, Meteors III (eds. C.-I. Lagerkvist, H. Rickman, B.A. Lindblad and M. Lindgren), University of Uppsala Press, Sweden, 571-574.

Whipple, F.L. and Hamid, S.E. (1952). 'On the origin of the Taurid meteor streams', Helwan Obs. Bull. No. 41, 1-30. 